\title{
Early postpartum resting-state functional connectivity for mothers receiving buprenorphine treatment for opioid use disorder: A pilot study
}

\author{
James E. Swain ${ }^{1,2}$ | S. Shaun $\mathrm{Ho}^{1}$
}

\author{
${ }^{1}$ Department of Psychiatry and Behavioral \\ Health \& Psychology, Stony Brook \\ University Medical Center, Stony Brook, \\ NY, USA \\ ${ }^{2}$ Department of Psychiatry, Psychology \\ and Center for Human Growth and \\ Development, University of Michigan, Ann \\ Arbor, MI, USA
}

\section{Correspondence}

James E. Swain, Department of Psychiatry, Stony Brook University Medical Center, HSC, T10-020, Stony Brook, NY 11794-8101, USA.

Email: james.swain@stonybrookmedicine. edu

Funding information National Center for Advancing Translational Sciences, Grant/Award Number:

ULT1TR00433; Stony Brook University

\begin{abstract}
Between 1999 and 2014, the prevalence of opioid use disorder (OUD) among pregnant women quadrupled in the USA. The standard treatment for peripartum women with OUD is buprenorphine. However, the maternal behavior neurocircuit that regulates maternal behavior and mother-infant bonding has not been previously studied for human mothers receiving buprenorphine treatment for OUD (BT). Rodent research shows opioid effects on reciprocal inhibition between maternal care and defence maternal brain subsystems: the hypothalamus and periaqueductal gray, respectively. We conducted a longitudinal functional magnetic resonance imaging (fMRI) pilot study in humans to specifically examine resting-state functional connectivity ( $r s-\mathrm{FC}$ ) between the periaqueductal gray and hypothalamus, as well as to explore associations with maternal bonding for BT. We studied 32 mothers who completed fMRI scans at 1 month (T1) and 4 months postpartum (T2), including seven mothers receiving buprenorphine for OUD and 25 non-OUD mothers as a comparison group (CG). The participants underwent a 6-minute resting-state fMRI scan at each time point. We measured potential bonding impairments using the Postpartum Bonding Questionnaire to explore how rs-FC with periaqueductal gray is associated with bonding impairments. Compared to CG, BT mothers differed in periaqueductal gray-dependent rs-FC with the hypothalamus, amygdala, insular cortex and other brain regions at $\mathrm{T} 1$, with many of these differences disappearing at $\mathrm{T} 2$, suggesting potential therapeutic effects of continuing buprenorphine treatment. In contrast, the "rejection and pathological anger" subscale of the Postpartum Bonding Questionnaire at T1 and T2 was associated with the T1-to-T2 increases in periaqueductal gray-dependent rs-FC with the hypothalamus and amygdala. Preliminary evidence links maternal bonding problems for mothers with OUD early in the postpartum to connectivity between specific care and defence maternal brain circuits, which may be mitigated by buprenorphine treatment. This exploratory study supports a potential mechanism for investigating both the therapeutic benefits and risks of opioids for maternal care and bonding with infants.
\end{abstract}

\section{KEYWORDS}

bonding, buprenorphine, hypothalamus, maternal brain behaviour neurocircuit, mothers, neuroimaging, opioid, periaqueductal gray, resting-state functional connectivity 


\section{1 | INTRODUCTION}

The opioid epidemic in the USA directly affects millions of women, with approximately $20 \%$ of all pregnant women being prescribed opioids and $2.5 \%$ chronically using opioids, ${ }^{1}$ and the number of pregnant women with opioid use disorder (OUD) having more than quadrupled from 1999 to 2014 (from 1.5 per 1,000 delivery hospitalisations to 6.5). ${ }^{2}$ The standard of care for OUD among pregnant women is opioid maintenance therapy with buprenorphine. Buprenorphine treatment for OUD (BT) ${ }^{3-6}$ is a common choice of treatment to mitigate the physiological stress associated with repeated cycles of maternal/foetal intoxication and withdrawal, preserving uterine stability via receptor occupancy. ${ }^{7-9}$ Compared to detoxification ${ }^{10,11}$ and possibly methadone treatment, ${ }^{12-20}$ BT may result in milder neonatal abstinence syndrome. ${ }^{6,21-28}$ However, the effects of buprenorphine treatment (BT) on the human maternal brain affected by OUD are not well understood. ${ }^{29}$ So far, there is just one study of mothers with polysubstance addictions showing reduced activation in reward regions of the brain in response to their own infant's face cues ${ }^{30}$; and one of mothers with BT for OUD vs depressed controls showing increased own vs other baby cry responses in multiple motivation brain regions. ${ }^{31}$

Maternal substance use in general places their infants at a fourfold increased risk for abuse or neglect, contributing to as much as $80 \%$ of child maltreatment cases ${ }^{32}$ and $60 \%$ of infant out-of-home placements. ${ }^{33}$ Opioid-induced deficits in homologous maternal behaviours have also been established in animal models. ${ }^{34-36}$ These effects appear to be mediated in part by activation of mu-opioid receptors in the hypothalamic medial preoptic area (mPOA). ${ }^{37-39}$ More recent findings have implicated the activation of select opioid receptor subtypes within the periaqueductal gray (PAG) in dysregulating maternal behaviour.

The PAG plays a pivotal role in switching from maternal caregiving behaviours to defensive/aggressive behaviours such as predation in rodents ${ }^{40}$ that are known to be regulated by mu and kappa opioid receptor activity. ${ }^{41,42}$ Indeed, infusion of a mu-opioid receptor agonist into the ventrolateral PAG disrupts the balance of maternal care and aggression and kappa-opioid receptor antagonism, shifting behaviours from maternal caring to predation behaviours in rodents. ${ }^{41}$ These recent results are particularly relevant for mothers receiving buprenorphine for OUD (BT) because buprenorphine is both a partial mu-opioid receptor agonist and kappa-opioid receptor antagonist. The hypothalamus and PAG are key parts of a maternal behaviour neurocircuit (MBN) (Figure 1) that is necessary for the survival of mammals from well-developed rodent models. ${ }^{43-49}$ In this scheme, maternal caregiving and defensive/aggressive systems are balanced with reciprocal inhibition of the hypothalamic mPOA and PAG respectively. ${ }^{50}$ Thus, mothers would not be distracted from sensitive maternal care in the absence of threat or exhibit insensitive or aggressive behaviour toward their infant.

We postulate that human mothers have a homologous MBN (Figure 1), ${ }^{51-53}$ including opposing maternal "care" and "defence" subsystems, that reciprocally inhibit each other to balance maternal sensitive infant care behaviours with need for defensive/aggressive behaviours that maintain safety. ${ }^{31}$ The care subsystem includes the mPOA in hypothalamus, ventral tegmental area, nucleus accumbens and ventral pallidum that regulate maternal caregiving behaviours such as licking and grooming for rodents, as well as sensitive, attuned care, gentle gaze, voice, touch and movement for humans. ${ }^{54}$ In the defence subsystem, the PAG may activate maternal defensive behaviours, such as aggression toward intruders and predatory behaviours. Among humans, PAG has been activated a baby distress cry, ${ }^{55}$ with such activity being related to mental state talk. ${ }^{56}$ This is interesting because a mother would need to balance caring and defensive behaviours in response to a cry which may solicit care or signal emergent concerns for safety. ${ }^{57}$ The regulation of other regions of the two subsystems of the MBN to orchestrate parenting has been demonstrated with human functional magnetic resonance imaging (fMRI) studies, including for the amygdala, ${ }^{58-60}$ insular cortex, $^{61,62}$ orbitofrontal cortex (OFC) ${ }^{63}$ and hypothalamus ${ }^{64-66}$ in response to infant cues. These regions may also be affected by

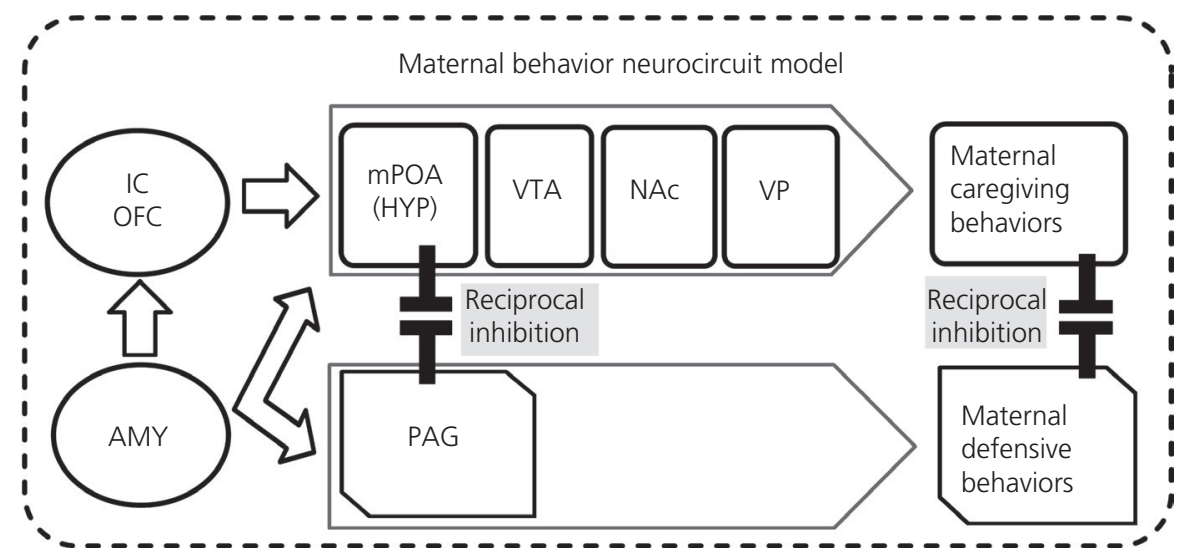

FIGURE 1 The maternal behavior neurocircuit (MBN) is comprised of two reciprocally inhibiting subsystems for: (1) maternal care, mediated by the medial preoptic area (mPOA) of hypothalamus (HYP), ventral tegmental area (VTA), nucleus accumbens (NAc) and ventral pallidum (VP), and (2) maternal defense, mediated by periaqueductal grey (PAG). These opposing subsystems are regulated by the amygdala (AMY), insula, and orbitofrontal cortex (OFC). Research from our lab and others has established that human MCN's volumes and responses to ethologically pertinent own-baby cry correlate with adaptive parental thoughts and behaviors 
addiction and polysubstance use, ${ }^{30,31,67,68}$ suggesting the need for more research on task-based brain physiology in the maternal brain. There is growing recognition, however, that high-order cognitive and emotional function, such as required for human parenting, may depend on coherent activity between key brain regions within neurocircuits, rather than single regions.

Resting-state functional connectivity ( $r$ - FC) is a method that is used in $\mathrm{fMRI}$ to examine regional interactions during a resting or task-negative state, during which time an explicit task is not being performed. ${ }^{69,70}$ Resting brain state conditions are observed through changes in blood-oxygen-level-dependent (BOLD) signals. Despite limitations of low temporal resolution and regional variation in neurovascular physiology, the resting state approach is useful to explore the brain's functional organisation in terms of apparent functional connectivity ${ }^{71}$ It has revealed networks of synchronous activity that are consistently found in healthy subjects, as well as in different stages of consciousness, in mental disorders and across species. ${ }^{72,73}$ As in the present study, rs-FC may be particularly to pursue a priori hypotheses in established circuits. ${ }^{74}$

So far, there are just one rodent and two human maternal brain studies using resting-state connectivity methods described above. Rat dams with early-life chronic social stress showed depressed maternal care specifically during early lactation, and changes in functional connectivity in regions associated with sensory processing, maternal and emotional responsiveness, memory and the reward pathway, including reduced PAG-anterior cingulate connectivity. ${ }^{75}$ Among human mothers, eight with depression vs nine healthy at 9 weeks postpartum, there was decreased functional connectivity in numerous corticolimbic circuits, such as coupling within the default mode network (DMN), including anterior cingulate cortex $(A C C)$, inferior parietal cortex and precuneus, as well as between DMN nodes and amygdala. ${ }^{76}$ In another human study performed at 10 weeks postpartum, 14 depressed vs 23 healthy mothers showed decreased DMN activity using a PCC seed region. ${ }^{77}$ These results may be interpreted to reflect diminished selfreflection and social cognition, ${ }^{78}$ as well as thoughts about duties and responsibilities to others, ${ }^{79}$ which are important to parenting. Additional research related to the content of maternal thoughts during the resting phase may be important to clarify the relationship between maternal thoughts moods and caregiving in health and mental illness. ${ }^{80}$

In the present study, we hypothesise that the specific functional connectivity between the hypothalamus and PAG, which are two functionally opposing regions in $\mathrm{MBN},{ }^{50}$ may be decreased in mothers receiving buprenorphine for OUD and relate to impaired bonding, consistent with rodent deficits in maternal behaviour with opioids. ${ }^{34,35}$ We thus examined rs-FC between the PAG and hypothalamus in BT mothers with reference to a comparison group (CG) of mothers who did not have OUD or receive any psychotrophic medication. Furthermore, to examine the roles of functional connectivity between PAG and hypothalamus in possible dysregulation of maternal behaviours, we also explored how the PAG-dependent rs-FC may be related to symptoms of mother-infant bonding impairments, as measured by the Postpartum Bonding Questionnaire (PBQ), ${ }^{81}$ in BT and CG mothers in the early postpartum period. The PBQ was chosen for this purpose because its subscales of Impaired Bonding (IB), Rejection and Pathological Anger (RPA) and Infant-Focused Anxiety (IFA) have been linked to maternal psychological abuse of infants and maternal depression in multiple cultures. ${ }^{82-85}$ We know that depression is common comorbidity of OUD ${ }^{86,87}$ and that depressive symptoms, even if not meeting the clinical threshold, ${ }^{88-90}$ can seriously risk adverse child outcomes across cognitive, behavioural and social domains. ${ }^{91-96}$

\section{2 | MATERIALS AND METHODS}

\section{1 | Procedures}

Participants were recruited from community health clinics, primary care clinics, and the University of Michigan Medicine Centers. They received an initial screening in the third trimester of pregnancy and completed questionnaires before undergoing two fMRI scanning sessions, at 1 month postpartum (T1) and 4 months postpartum (T2). Participants with OUD were prescribed BT as part of routine medical care and there were no infant admissions for medical complications after initial neonatal abstinence syndrome. All procedures were approved by the Human Subject Institutional Review Board at University of Michigan.

\section{2 | Participants}

For the sample in the present study ( $n=32$ ), we included group of mothers who were maintained on buprenorphine replacement therapy for their OUD (BT) $(n=7$, years of age; mean \pm SD $29.86 \pm 6.62$ years) and a comparison group (CG) of mothers who reported no opioid or other substance uses or any psychotrophic medications (CG, $n=25$, years of age; mean \pm SD $27.62 \pm 8.46$ years). Mean \pm SD ages for infants of early postpartum mothers were $0.08 \pm 0.01$ years for BT and $0.07 \pm 0.27$ years for CG. The BT mothers received buprenorphine medication during pregnancy and continued throughout the time of the study. The mean \pm SD dose recorded every 2 weeks postpartum was $14.18 \pm 3.35 \mathrm{mg}$, with six out of seven mothers stabilising between 12 and $16 \mathrm{mg}$.

\section{3 | Self-reported measure}

\subsection{1 | Postpartum bonding questionnaire}

The PBQ is a 25-item self-report questionnaire developed to screen early postpartum women for potential parent-infant bonding disorders. ${ }^{97}$ The PBQ consists of four subscales to indicate factors of clinical relevance, namely IB (subscale 1 with sensitivity of 0.82 for detecting mothers with bonding disorders) (e.g. "I feel distant from my baby"); RPA (subscale 2 with sensitivity of 0.88 for infant rejection and 0.67 for severe anger), identifies mothers with serious infant-oriented hostility (e.g. "I resent my baby"); IFA (subscale 3), 
which may be useful in anxious mothers (e.g. "My baby makes me feel anxious"); and subscale 4, which signals the presence of incipient abuse that would require urgent intervention (e.g. "I have done harmful things to my baby"). None of the participants indicated abuse from subscale 4 and only the first three subscales (IB, RPA and IFA) were examined in the current study.

\section{4 | Functional and structural magnetic resonance imaging}

\subsection{1 | Imaging data acquisition}

During the fMRI scanning session, each participant was positioned in a supine orientation with her head positioned in a head coil. Visual stimuli (a cross at the centre) were presented via a goggle system and NordicNeuroLab (Bergen, Norway) audio system. The fMRI scanning was performed with a 3.0 Tesla Philips magnetic resonance imaging scanner (Philips, Eindhoven, The Netherlands) using a standard eight-channel radiofrequency SENSE head coil. with the following acquisitions: (i) a high-resolution T1 scan was acquired to provide precise anatomical localisation (TR of $9.8 \mathrm{~ms}, \mathrm{TE}=459 \mathrm{~ms}, \mathrm{FA}=8^{\circ}$, FOV of $256 \mathrm{~mm}$, slice thickness of $1.0 \mathrm{~mm}, 180$ slices with $288 \times 288$ matrix per slice) and (ii) A run of T2*-weighted BOLD echo planar imaging sequence volumes was acquired for whole-brain fMRI during the resting-state task $(180$ frames, $T R=2000 \mathrm{~ms}, \mathrm{TE}=30 \mathrm{~ms}$, $\mathrm{FA}=90^{\circ}, \mathrm{FOV}=220 \mathrm{~mm}, 42$ contiguous axial slices, slice thickness $=2.8 \mathrm{~mm}$ with $64 \times 64$ matrix per slice, voxel size $=3.44 \times 3.44$ $\times 2.8 \mathrm{~mm}^{3}$ ), after discarding the first five initial frames to allow for equilibration of scanner signal.

TABLE 1 Demographics

\begin{tabular}{|c|c|c|}
\hline & BT & CG \\
\hline \multicolumn{3}{|l|}{ Age } \\
\hline Mean & 29.86 & 27.62 \\
\hline SD & 6.62 & 8.46 \\
\hline \multicolumn{3}{|l|}{ Infant age at T1 scan } \\
\hline Mean & 0.08 & 0.07 \\
\hline SD & 0.01 & 0.027 \\
\hline \multicolumn{3}{|l|}{ Number of child } \\
\hline Mean & 1.71 & 1.60 \\
\hline $\mathrm{SD}$ & 0.76 & 0.66 \\
\hline \multicolumn{3}{|l|}{ Race } \\
\hline European American & 6 & 13 \\
\hline African American & 0 & 12 \\
\hline Native American & 1 & 0 \\
\hline \multicolumn{3}{|l|}{ Social economic status } \\
\hline Low (under $\$ 20 \mathrm{~K}$ household income) & 4 & 14 \\
\hline Middle & 3 & 11 \\
\hline
\end{tabular}

Abbreviations: BT, buprenorphine treatment for opioid use disorder; $\mathrm{CG}$, comparison group.

\subsubsection{Imaging data processing and analysis}

Functional MRI data were pre-processed and analysed using statistical parametric mapping software (spM8; Welcome Department of Imaging Neuroscience, London, UK). Slice timing correction was performed using a middle slice as a reference (slice 21). ${ }^{98}$ After slice time correction, images within each run were realigned to the mean image of the first run to correct for movement. Realigned functional images and structural images were spatially normalised using the DARTEL method in SPM8. ${ }^{99}$ The normalised functional images were re-sliced to $2 \times 2 \times 2 \mathrm{~mm}$ voxels. Images were then spatially smoothed using a Gaussian filter with a full-width half-maximum value of $8 \mathrm{~mm}$. To assess and mitigate movement artifact, we calculated the frame-wise displacement (FD), defined as the sum (in millimetres) of the absolute values of frame displacement in successive functional volumes, based on six translation and rotation parameters from the realignment file. ${ }^{100}$ Rotational displacements were converted from degrees to millimeters moved on the surface of a 50-mm sphere. A frame-scrubbing routine was used to censor out any frame with FD $>0.5 \mathrm{~mm}$ from the regression analysis described below, yielding a scrub ratio for each subject, with a cut-off of $60 \%$ censored-out frames for sample exclusion, in accordance with the literature showing that as many as $60 \%$ of frames can be removed and still yield analysable results. ${ }^{100,101}$ There was no BT vs CG difference in the averaged FD before scrubbing (BT: mean \pm SD: $0.379 \pm 0.063$; CG: mean \pm SD: $0.300 \pm 0.028$; $\left.F_{1,40}=1.284, \mathrm{MS}_{\text {error }}=0.056, P=.264\right)$. This suggests no significant head movement as a potential confounding factor between groups. Three-compartment segmentation of the high-resolution structural image from the DARTEL normalisation were applied to the functional time series to extract cerebral spinal volume and white matter compartments, which then were subjected to a principal component analysis to identify the top five components in each, ${ }^{102}$ which should correspond to heart rate and respiratory effects on the BOLD signal. ${ }^{103}$ Adjusted time courses were derived from sequential regressions of the time series with the following regressors: linear trend of six motion parameters, their temporal derivatives, the quadratics of these 12 parameters, five components from the principal components analysis of cerebral spinal volume, five components of white matter, followed by band-pass filtering from 0.01 to $0.1 \mathrm{~Hz}$, and then motion scrubbing. The seed mask of PAG, as a region of interest (ROI), in rsfMRI analysis was generated using PICKATLAS software (WFU PickAtlas; http://fmri.wfubmc.edu/software/pickatlas) as a box-shaped mask centered at the ventrolateral PAG (MNI: $x=0, y=-27, z=-8$, with $3 \times 1 \times 1 \mathrm{~mm}$ extensions), in accordance with the literature. ${ }^{104,105}$ In the first-level analysis, adjusted time courses were averaged across all voxels in the seed mask. The average time course in the seed was correlated with time courses from all other voxels in the brain, Fisher $r$-to-Z transformed, and taken to second level, between-group analyses. In the second-level analysis, statistical parametric maps in the first model were submitted to general linear models (GLMs) to test for the effects of interest. The effects of interest included the group difference (BT vs Controls) in the sample of 32 mothers and each of the three PBQ subscales in a subsample of BT and CG mothers $(n=5$ in 

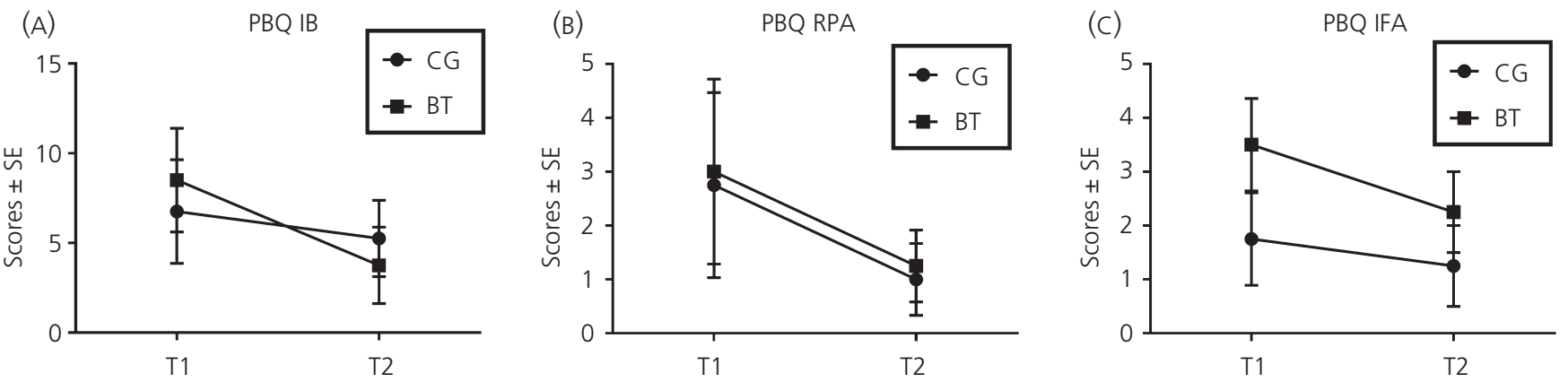

FIGURE 2 The group by timeline charts for three subscales of postpartum bonding questionnaire (PBQ). A, The impaired bonding subscale (IB). B, The rejection and pathological anger subscale (RPA). C, The infant-focused anxiety subscale (IFA). Abbreviations: BT, buprenorphine treatment for opioid use disorder; CG, comparison group. T1, 1 month postpartum; T2, 4 months postpartum

TABLE 2 BT vs CG main effects on PAG-dependent rs-FC at T1

\begin{tabular}{|c|c|c|c|c|c|c|}
\hline \multirow[b]{2}{*}{ Brain region } & \multirow[b]{2}{*}{ Side } & \multicolumn{3}{|c|}{ MNI coordinates } & \multirow{2}{*}{$\begin{array}{l}\text { Number } \\
\text { of voxels }\end{array}$} & \multirow[b]{2}{*}{ Z score } \\
\hline & & $x$ & y & z & & \\
\hline \multicolumn{7}{|l|}{$\mathrm{BT}>\mathrm{CG}$} \\
\hline Inferior temporal gyrus & $\mathrm{R}$ & 68 & -28 & -24 & 119 & 5.21 \\
\hline Septal area/caudate nucleus & $R / L$ & -4 & 16 & 4 & 478 & 5.05 \\
\hline Amygdala/hippocampus & $\mathrm{R}$ & 24 & -14 & -12 & 220 & 4.88 \\
\hline Hypothalamus $^{a}$ & $\mathrm{R} / \mathrm{L}$ & 4 & -2 & -12 & 44 & 2.42 \\
\hline \multicolumn{7}{|l|}{$\mathrm{BT}<\mathrm{CG}$} \\
\hline Supramarginal gyrus & $\mathrm{R}$ & 58 & -46 & 30 & 1111 & 5.55 \\
\hline Middle temporal gyrus & L & -46 & -58 & 8 & 487 & 5.14 \\
\hline $\begin{array}{l}\text { Posterior cingulate cortex/ } \\
\text { precuneus }\end{array}$ & $\mathrm{R}$ & 14 & -46 & 12 & 655 & 4.94 \\
\hline Postcentral gyrus & L & -68 & -14 & 26 & 108 & 4.61 \\
\hline Middle cingulate cortex & $\mathrm{R}$ & 18 & -36 & 40 & 298 & 4.59 \\
\hline Insular cortex & $\mathrm{R}$ & 48 & 6 & -2 & 122 & 4.57 \\
\hline Precentral gyrus & $\mathrm{R}$ & 48 & -2 & 50 & 86 & 4.51 \\
\hline Precuneus, dorsal & $\mathrm{R} / \mathrm{L}$ & -6 & -56 & 60 & 160 & 4.49 \\
\hline
\end{tabular}

Abbreviations: BT, buprenorphine treatment for opioid use disorder; CG, comparison group; PAG, periaqueductal gray; rs-FC, resting-state functional connectivity.

${ }^{a}$ Except this a priori region of interest, all regions listed above were cluster-level whole-brain corrected (family-wise error correction <.05) at voxel-wise $P=.001$. each group). All of the second-level models were controlled for wholebrain multiple comparisons at the cluster level for voxel-wise intensity threshold at $P<.001$, which is considered to yield reliable statistical inference. ${ }^{106}$ We also used Threshold-Free Cluster Enhancement (TFCE) method 107 to perform family-wise small volume correction (s.v.c.) in a ROI mask of the hypothalamus (MNI: $x= \pm 4, y=-2, z=-12$, with a 4-mm radius) to examine the PAG-hypothalamus functional connectivity in accordance with the hypothesis that the reciprocal inhibition between PAG and hypothalamus plays a key role with respect to regulating maternal behaviours.

\section{5 | Behavioural data analysis}

We investigated the associations between the PAG-hypothalamus functional connectivity and three subscales of PBQ (IB, RPA and
IFA) at T1 and T2 in the early postpartum mothers, using bivariate Pearson's $r$ correlation analysis in SPSs, version 24 (IBM Corp., Armonk, NY, USA).

\section{3 | RESULTS}

\section{1 | Behavioural results}

The participants in BT and CG did not differ with respect to their age $\left(F_{1,30}=0.081, \mathrm{MS}_{\text {error }}=70.25, P=.78\right)$, number of offspring $\left(F_{1,30}=0.16, \mathrm{MS}_{\text {error }}=0.45, P=.69\right)$ and youngest child's age in the early postpartum mothers $\left(F_{1,8}=1.00, \mathrm{MS}_{\text {error }}=0.01\right.$, $P=.35$ ). For demographics, see Table 1 . In a mixed model of GLM, we investigated the main effects of group (BT vs CG), Time (T1 and T2) and Time by Group interaction effect in three subscales 
T1 Group main effects on rs-FC w/PAG
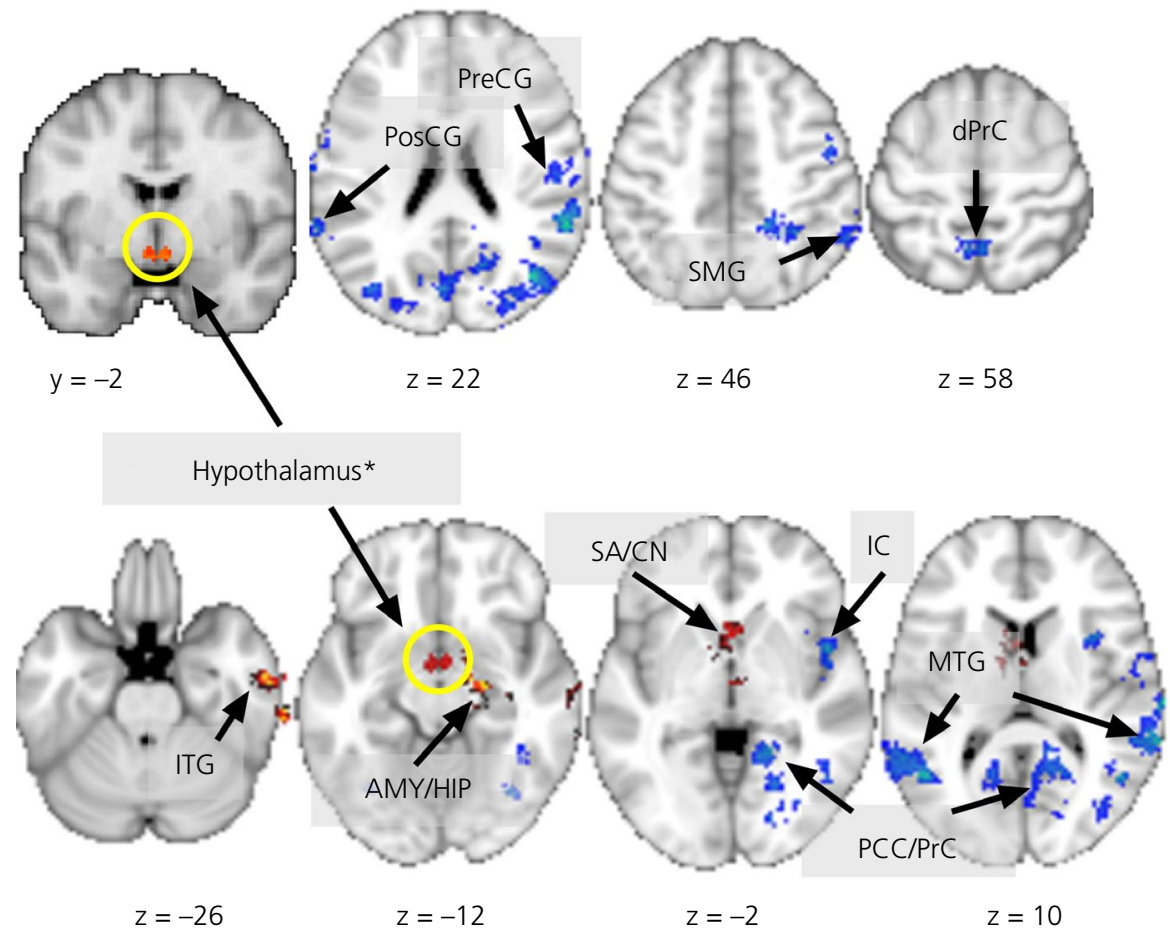

FIGURE 3 The group main effects on the periaqueductal gray (PAG)-dependent resting-state functional connectivity (rs-FC) at 1 month postpartum. The red/warm coloured clusters indicate buprenorphine treatment for opioid use disorder (BT) > comparison group (CG) differences and the blue/winter colored clusters indicate CG > BT differences; all clusters shown are whole-brain corrected at the cluster level, except the a prior region of interest, hypothalamus*. Abbreviations: AMY/HIP, amygdala/hippocampus; dPrC, dorsal precuneus; IC, insular cortex; ITG, inferior temporal gyrus; MTG, middle temporal gyrus; PCC/Prc, posterior cingulate cortex/precuneus; PosCG, postcentral gyrus; PreCG, precentral gyrus; SA/CN, septal area/caudate nucleus; SMG, supramarginal gyrus. The z- or $y$-coordinates of the slices are listed below the images, which are rendered in neurological convention (the right side of image is the right hemisphere)

\begin{tabular}{|c|c|c|c|c|c|c|}
\hline \multirow[b]{2}{*}{ Brain region } & \multirow[b]{2}{*}{ Side } & \multicolumn{3}{|c|}{ MNI coordinates } & \multirow[b]{2}{*}{ Number of voxels } & \multirow[b]{2}{*}{ Z score } \\
\hline & & $x$ & y & $z$ & & \\
\hline \multicolumn{7}{|l|}{$\mathrm{BT}>\mathrm{CG}$} \\
\hline Hypothalamus $^{a}$ & $R / L$ & -8 & -2 & -12 & 5 & 2.01 \\
\hline \multicolumn{7}{|l|}{$\mathrm{BT}<\mathrm{CG}$} \\
\hline $\begin{array}{l}\text { Anterior cingulate } \\
\text { cortex, dorsal }\end{array}$ & $R / L$ & 2 & 32 & 2 & 198 & 4.48 \\
\hline $\begin{array}{l}\text { Anterior cingulate } \\
\text { cortex, ventral }\end{array}$ & L & -12 & 42 & -10 & 89 & 3.82 \\
\hline
\end{tabular}

TABLE 3 BT vs CG main effects on PAG-dependent rs-FC at T2

Abbreviations: BT, buprenorphine treatment for opioid use disorder; CG, comparison group; PAG, periaqueductal gray; rs-FC, resting-state functional connectivity.

${ }^{a}$ Except this a priori region of interest, all regions listed above were cluster-level whole-brain corrected (family-wise error correction $<.05$ ) at voxel-wise $P=.001$.

of PBQ. We found that there were no significant group main effects for any of the PBQ subscales (IB: $F_{1,6}=0.002, \mathrm{MS}_{\text {error }}=35.90$, $P=.97 ;$ RFA: $F_{1,6}=0.026, \mathrm{MS}_{\text {error }}=9.63, P=.88 ;$ IFA: $F_{1,6}=1.49$, $\mathrm{MS}_{\text {error }}=5.06, P=.27$ ); there was a significant main effect of Time in IFA $\left(F_{1,6}=21.00, \mathrm{MS}_{\text {error }}=0.15, P=.004\right)$, although not the other subscales (IB: $F_{1,6}=2.45, \mathrm{MS}_{\text {error }}=15.98, P=.17$; RPA: $F_{1,6}=3.10$,
$\mathrm{MS}_{\text {error }}=3.96, P=.13$ ); there were no significant Time by Group interaction effects for any of the subscales, although there was a marginal trend for IFA (IB: $F_{1,6}=0.066, \mathrm{MS}_{\text {error }}=15.98, P=.45$; RFA: $F_{1,6}=0.001, \mathrm{MS}_{\text {error }}=3.96, P=1.00 ;$ IFA: $F_{1,6}=3.86, \mathrm{MS}_{\text {error }}=0.15$, $P=.10$ ). For the Time by Group line charts of PBQ subscales, see Figure 2. 


\section{2 | Neuroimaging results}

\subsubsection{Main effects of group at T1 and T2, time, and time by group interactions}

We examined the main effects of Group at timepoint, the main effects of Time and the Time by Group interaction effects on the PAGdependent resting-state functional connectivity ( $r s-F C$ ) in SPM8. There were no Time main effects observed in the PAG-dependent rs-FC.

The main effects of Group (BT vs CG) on PAG-dependent rs-FC at T1 are summarised in Table 2 and Figure 3. At T1, BT vs CG mothers showed greater PAG-dependent rs-FC in the right inferior temporal gyrus, dorsal caudate nucleus/septal area and right amygdala/ hippocampus complex; when examining a prior ROI using TFCE, we also found that the PAG-dependent rs-FC with the hypothalamus was stronger in BT compared to CG mothers at T1(MNI: [4, -4, -10], 25 voxels, TFCE $=27.00, P=.026$ s.v.c.). Conversely, BT vs CG mothers showed a less PAG-dependent $r s-F C$ in the right supramarginal gyrus, bilateral middle temporal gyrus (MTG), precuneus/posterior cingulate cortex, bilateral pericentral gyrus and right insular cortex at $\mathrm{T} 1$.

The main effects of Group (BT vs CG) on PAG-dependent rs-FC at T2 are summarised in Table 3 and Figure 4. At T2, BT mothers showed PAG-dependent rs-FC that was greater than those in CG mothers in the hypothalamus ROI (MNI: $[-6,-4,-10]$, three voxels, $\mathrm{TFCE}=19.61, P=.041$ s.v.c.). Conversely, BT mothers showed a less PAG-dependent $\mathrm{rs}-\mathrm{FC}$ in the dorsal and ventral ACC (dACC and vACC).
For the Time by Group interaction effects, the results are summarised in Table 4 and Figure 5. We found that, compared to CG mothers, BT mothers showed greater T1-to-T2 increases of the PAGdependent rs-FC with the right MTG and insular cortex; conversely, there were greater T1-to-T2 decreases in the PAG-dependent rs-FC with the dorsal precuneus, left OFC, septal area and dACC.

\subsection{2 | PBQ associations with PAG-dependent rs-FC}

We explored the associations between three subscales of $\mathrm{PBQ}$ (IB, RPA and IFA) and the PAG-dependent rs-FC with the brain regions reported in the results above in the early postpartum mothers, namely the hypothalamus, right amygdala/hippocampus, right MTG, right insular cortex, septal area/caudate nucleus, dACC, left OFC and dorsal precuneus (for the line charts of the parameter estimates of these regions by group and time, see Figure 6). Among the regions reported above, only the hypothalamus and right amygdala PAG-dependent rs-FC were associated with some of the subscales in $\mathrm{PBQ}$, as described below. The BT and CG mothers did not differ with respect to the associations between any of the $\mathrm{PBQ}$ subscales and PAG-dependent rs-FC.

Across both groups, the T1-to-T2 changes in the PAG-dependent $\mathrm{rs}-\mathrm{FC}$ with the hypothalamus were associated with T1 PBQ IB $(r=.70, P=.025)$ and RPA $(r=.76, P=.011)$, but not IFA $(r=.31$, $P=.38)$, and with T2 PBQ RPA $(r=.81, P=.014)$, but not IB $(r=.46$, $P=.25)$ and IFA ( $r=-.097, P=.82)$ (Figure 7). The T1-to-T2 changes in the PAG-dependent $r s-F C$ with the right amygdala were associated with T1 PBQ RPA $(r=.72, P=.019)$ and IFA $(r=.65, P=.043)$, but not

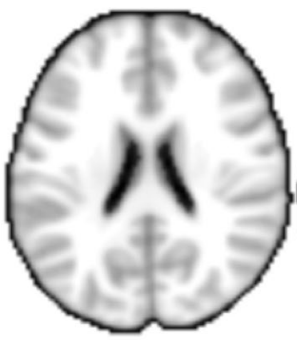

$$
z=22
$$

$$
z=34
$$

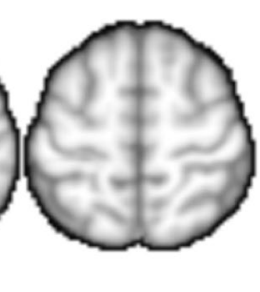

$z=58$ resting-state functional connectivity (rs-FC) at 4 months postpartum. The blue/winter colored clusters indicate comparison group (CG) > buprenorphine treatment for opioid use disorder (BT) differences, all clusters shown are whole-brain corrected at the cluster level. Abbreviations: dACC, dorsal anterior cingulate cortex; $\mathrm{VACC}$, ventral anterior cingulate cortex. The $z$-coordinates of the slices are listed below the images, which are rendered in neurological convention (the right side of image is the right hemisphere)

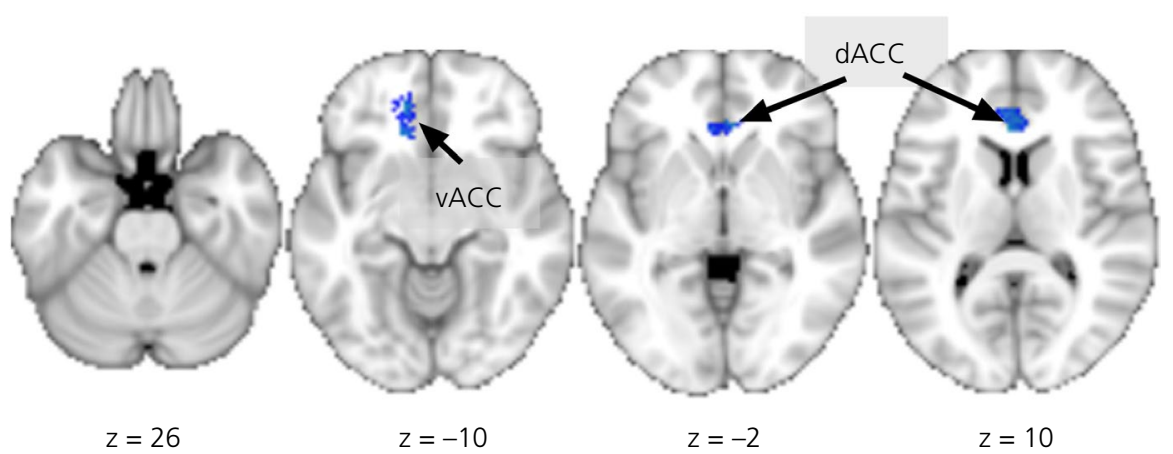




\begin{tabular}{|c|c|c|c|c|c|c|}
\hline \multirow[b]{2}{*}{ Brain region } & \multirow[b]{2}{*}{ Side } & \multicolumn{3}{|c|}{ MNI coordinates } & \multirow[b]{2}{*}{ Number of voxels } & \multirow[b]{2}{*}{$Z$ score } \\
\hline & & $x$ & y & z & & \\
\hline \multicolumn{7}{|c|}{ BT > CG in T1-to-T2 increases } \\
\hline $\begin{array}{l}\text { Middle temporal } \\
\text { gyrus }\end{array}$ & $\mathrm{R}$ & 60 & -40 & 4 & 211 & 4.78 \\
\hline Insular cortex & $\mathrm{R}$ & 48 & 10 & -6 & 110 & 4.51 \\
\hline \multicolumn{7}{|c|}{ BT > CG in T1-to-T2 decreases } \\
\hline Precuneus, dorsal & L & -26 & -74 & 50 & 137 & 5.03 \\
\hline $\begin{array}{l}\text { Orbital frontal } \\
\text { cortex }\end{array}$ & L & -18 & 32 & -18 & 169 & 4.84 \\
\hline $\begin{array}{l}\text { Septal area/cau- } \\
\text { date nucleus }\end{array}$ & $R / L$ & -10 & 0 & 6 & 271 & 4.78 \\
\hline $\begin{array}{l}\text { Anterior cingulate } \\
\text { cortex, dorsal }\end{array}$ & L & -2 & 30 & 10 & 175 & 4.58 \\
\hline
\end{tabular}

TAB LE 4 Group (BT vs CG) by Time interaction effects on PAG-dependent rs-FC

Note: All regions listed above were cluster-level whole-brain corrected (family-wise error correction <.05) at voxel-wise $P=.001$.

Abbreviations: BT, buprenorphine treatment for opioid use disorder; CG, comparison group; PAG, periaqueductal gray; rs-FC, resting-state functional connectivity.

FIGURE 5 The group by time interaction effects on the periaqueductal gray (PAG)-dependent resting-state functional connectivity ( $r$-FC). The $\mathrm{red} /$ warm colored clusters indicate buprenorphine treatment for opioid use disorder (BT) > comparison group (CG) in 1 month postpartum (T1) to 4 months postpartum (T2) increases and the blue/ winter colored clusters indicate BT > CG in T1-to-T2 decreases, all clusters shown are whole-brain corrected at the cluster level. Abbreviations: dACC, dorsal anterior cingulate cortex; IC, insular cortex; MTG, middle temporal gyrus; SA/CN, septal area/caudate nucleus; OFC, orbital frontal cortex. The $z$-coordinates of the slices are listed below the images, which are rendered in neurological convention (the right side of image is the right hemisphere)
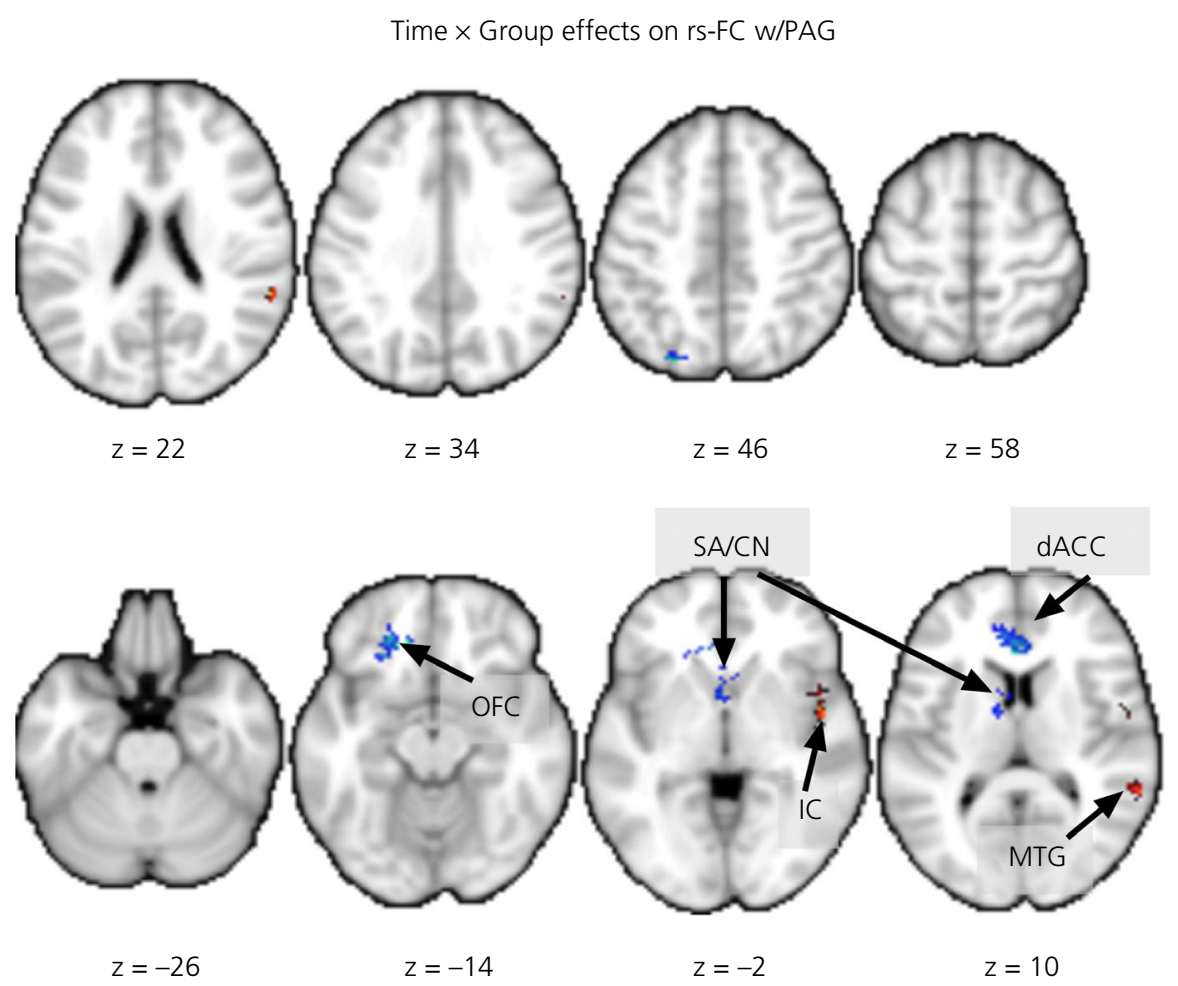

IB $(r=.48, P=.17)$, and with T2 PBQ IB $(r=.82, P=.013)$ and RPA $(r=.73, P=.039)$, but not IFA $(r=.41, P=.32)$ (Figure 8$)$.

\section{4 | DISCUSSION}

In the context of an unprecedented opioid crisis in the USA, many peripartum women with OUD are treated with buprenorphine maintenance treatment, ${ }^{3}$ despite concerns for parenting among affected human mothers. ${ }^{29}$ Rodent research indicates that opioids can disrupt maternal behaviour by acting on the PAG and other maternal brain circuit regions including the hypothalamus. ${ }^{34-36} \mathrm{We}$ investigated whether a group of women with OUD and buprenorphine treatment (BT mothers) differed from a comparison group (CG) of non-OUD mothers during the early postpartum with respect to the resting-state functional connectivity associated with the PAG (PAG-dependent $r s-F C$ ) in the maternal brain neurocircuit. ${ }^{48,49}$ We found that, at 1 month postpartum (T1), compared to CG, BT mothers showed greater PAG-dependent rs-FC in the right inferior temporal gyrus, septal area/caudate nucleus, right 
FIGURE 6 The group by timeline charts for brain regions showing group differences in their resting-state functional connectivity ( $\mathrm{rs}-\mathrm{FC}$ ) with the periaqueductal gray (PAG), as indicated in Figures 3-5. A, Hypothalamus. B, Right amygdala/hippocampus. C, Right middle temporal gyrus. D, Right insular cortex. E, Septal area/caudate nucleus. F, Dorsal anterior cingulate cortex. G, Left orbital frontal cortex. H, Dorsal precuneus. Abbreviations: BT, buprenorphine treatment for opioid use disorder; CG, comparison group. T1, 1 month postpartum; T2, 4 months postpartum

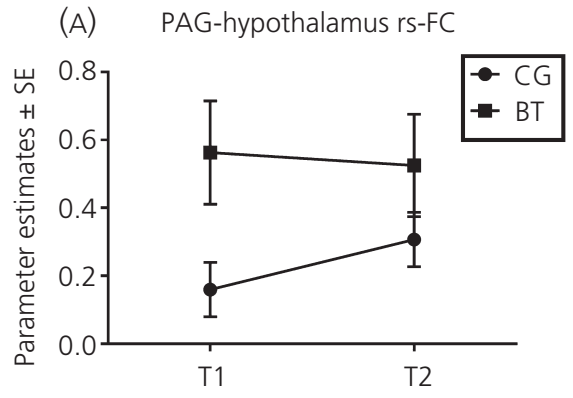

(B) PAG-R Hipp/Amy rs-FC

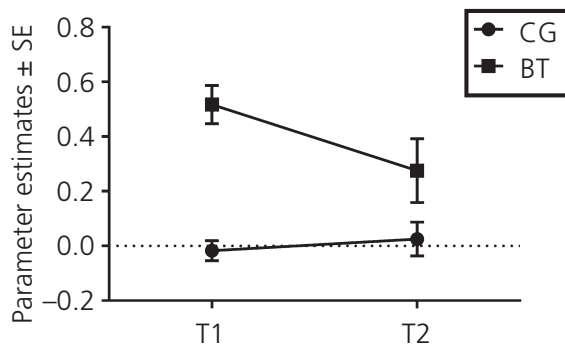

(C) PAG-R MTG rs-FC

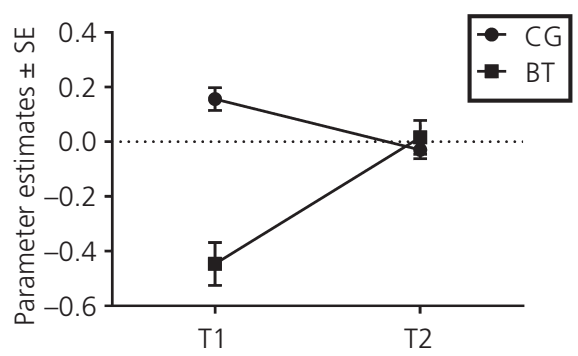

(D)

PAG-R Insula rs-FC

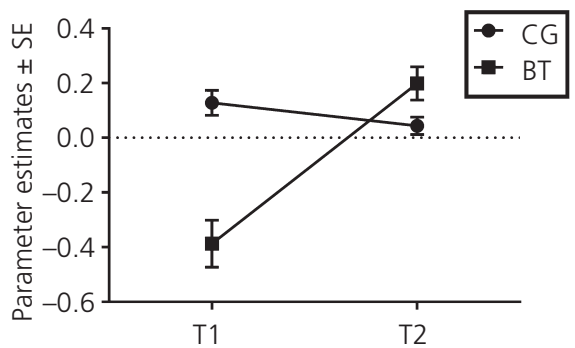

(E)

PAG-SA/CN rs-FC

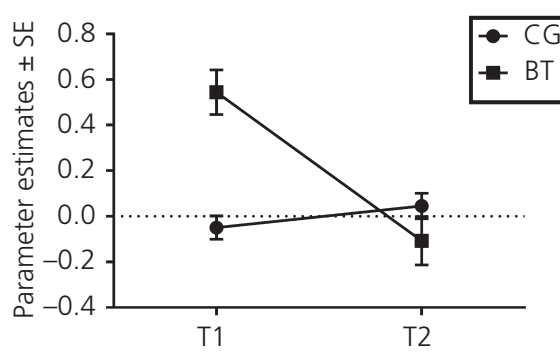

(F)

PAG-dACC rs-FC

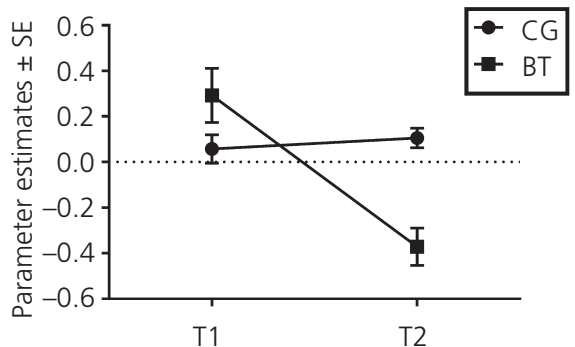

(G)

PAG-L OFC rs-FC

$(\mathrm{H})$

PAG-dPrecuneus rs-FC

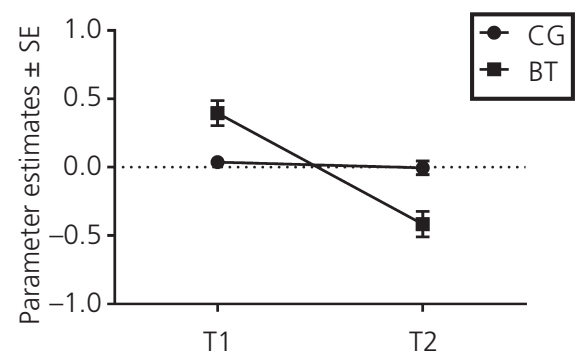

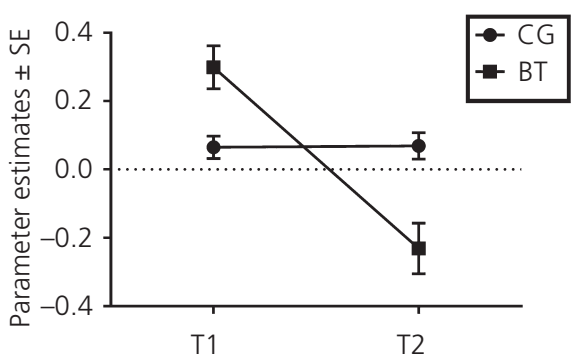

amygdala/hippocampus and hypothalamus; they also showed a less PAG-dependent rs-FC in the supramarginal gyrus, posterior cingulate cortex/precuneus, postcentral gyrus, middle cingulate cortex, insular cortex, precentral gyrus and dorsal precuneus. At 4 months postpartum (T2), compared to CG, BT mothers showed a marginally greater PAG-dependent $r s-F C$ in the hypothalamus and a significantly less PAG-dependent rs-FC in the ventral and dorsal anterior cingulate cortex. In terms of Time by Group interaction, BT mothers, compared to CG, showed greater T1-to-T2 increases in the PAG-dependent $r s-F C$ in the right middle temporal gyrus and insular cortex as a result of a reversal of the initial negative rsFC with PAG in these regions; they also showed greater T1-to-T2 decreases in the PAG-dependent rs-FC in the septal area/caudate nucleus, dorsal anterior cingulate cortex, left orbital frontal cortex and dorsal precuneus as a result of a reversal of the initial positive rs-FC with PAG in these regions.

We also explored whether the PAG-dependent rs-FC in any of the brain regions, showing group differences, was associated with deficits in maternal behaviours, as measured by the PBQ. Although the BT and CG mothers did not differ with respect to the subscales of PBQ, such as for IB, RPA and IFA, we found that the T1-to-T2 changes in PAG-dependent rs-FC with the hypothalamus and right amygdala were positively correlated with some of the subscales of PBQ at T1 and T2. By contrast to group difference 
(A)

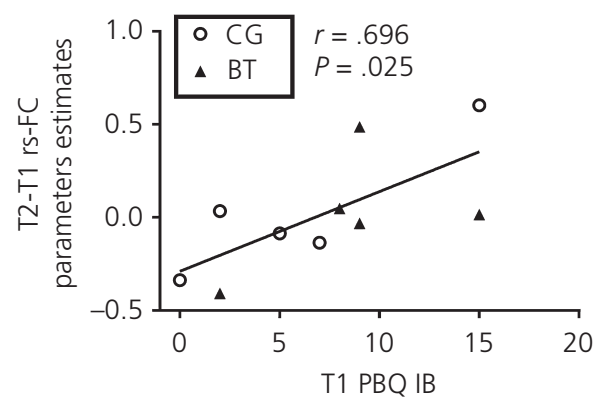

(c)

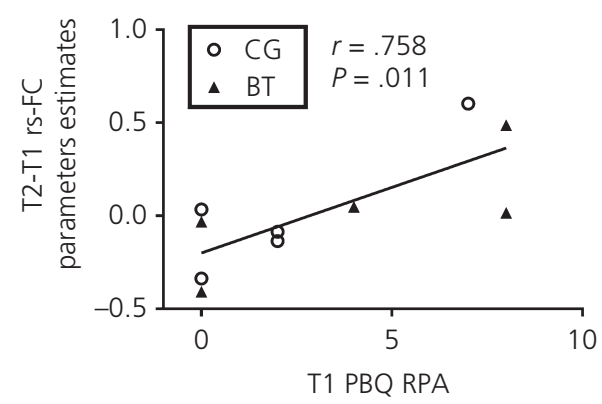

(E)

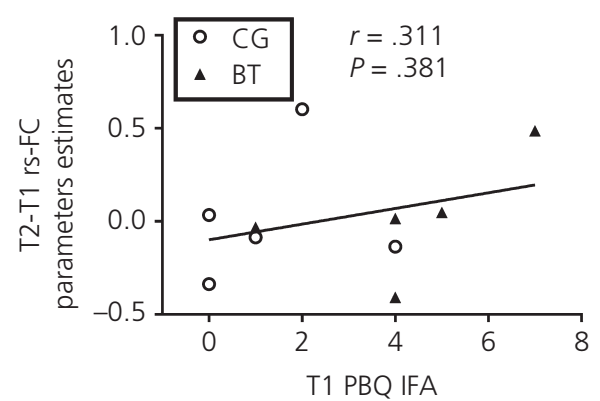

(B)

PAG-hypothalamus rs-FC

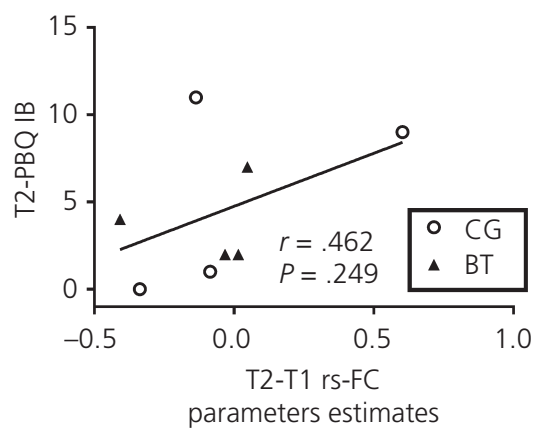

(D) PAG-hypothalamus rs-FC

(F) PAG-hypothalamus rs-FC
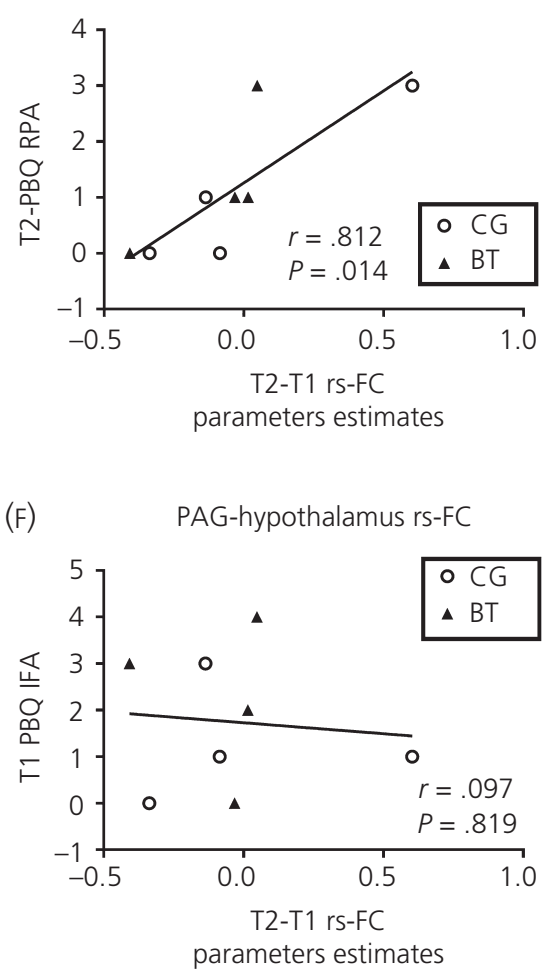

FIGURE 7 The scatter plots relating the 1 month postpartum (T1) to 4 months postpartum (T2) changes in the periaqueductal gray (PAG)-hypothalamus resting-state functional connectivity (rs-FC) to T1/T2 Postpartum Bonding Questionnaire (PBQ) subscales of impaired bonding (IB) (A, B), rejection and pathological anger (RPA) (C, D) and infantfocused anxiety (IFA) (E, F). Abbreviations: BT, buprenorphine treatment for opioid use disorder; CG, comparison group analysis, correlation analysis may have detected a difference because of the increased power resulting from data pooling across BT and CG groups. For the PAG-hypothalamus rs-FC, the IB and RPA (but not IFA) at T1 were correlated with the T1-to-T2 increases in the PAG-hypothalamus rs-FC; furthermore, the T1-to-T2 increases in the PAG-hypothalamus rs-FC were correlated with the RPA (but not IB and IFA) at T1. For the PAG-amygdala rs-FC, the RPA and IFA (but not IB) at T1 were correlated with the T1-to-T2 increases in the PAG-amygdala rs-FC; furthermore, the T1-to-T2 increases in the PAG-amygdala rs-FC were correlated with the IB and RPA (but not (FA) at T1. In sum, we found that, among the three subscales of PBQ, RPA was related to the PAG-dependent rs-FC to the greatest extent. Because RPA reflect outward expression of maternal aggression towards the infants, we have found preliminary evidence for the roles of the PAG-hypothalamus and PAG-amygdala rs-FC in the dysregulation of maternal aggression, which may reflect an impairment in the reciprocal inhibition between PAG and the hypothalamus and their roles in maternal aggression and caregiving behaviours, respectively.

Among human mothers, PAG regional responses have been related to observing pictures of a mother's own child vs an unknown child. ${ }^{108,109}$ These task-based studies have not analysed the reciprocal inhibition between PAG and maternal care regions such as the hypothalamus. It is possible that PAG activation in these studies was part of a balanced response to own infant cues, in which mothers may have been preparing to exhibit caring or defensive behaviours depending on other circumstances. In support, in a recent study of mothers, ${ }^{110}$ PAG connectivity with OFC during own vs other baby picture task was inversely related to parenting stress. Perhaps, in that study, ${ }^{110}$ stress reduces OFC regulation of defensive and aggressive drives from the PAG, in accordance with circumstances in which a stressed mother must prepare to defend their infant. Future studies may be able to assess the timing of regional brain activity, aiming to describe the order by which brain regions influence each 
FIGURE 8 The scatter plots relating the 1 month postpartum (T1) to 4 months postpartum (T2) changes in the periaqueductal gray (PAG)-right amygdala resting-state functional connectivity (rs-FC) to T1/T2 Postpartum Bonding Questionnaire (PBQ) subscales of impaired bonding (IB) (A, B), rejection and pathological anger (RPA) (C, D) and infantfocused anxiety (IFA) (E, F). Abbreviations: BT, buprenorphine treatment for opioid use disorder; CG, comparison group
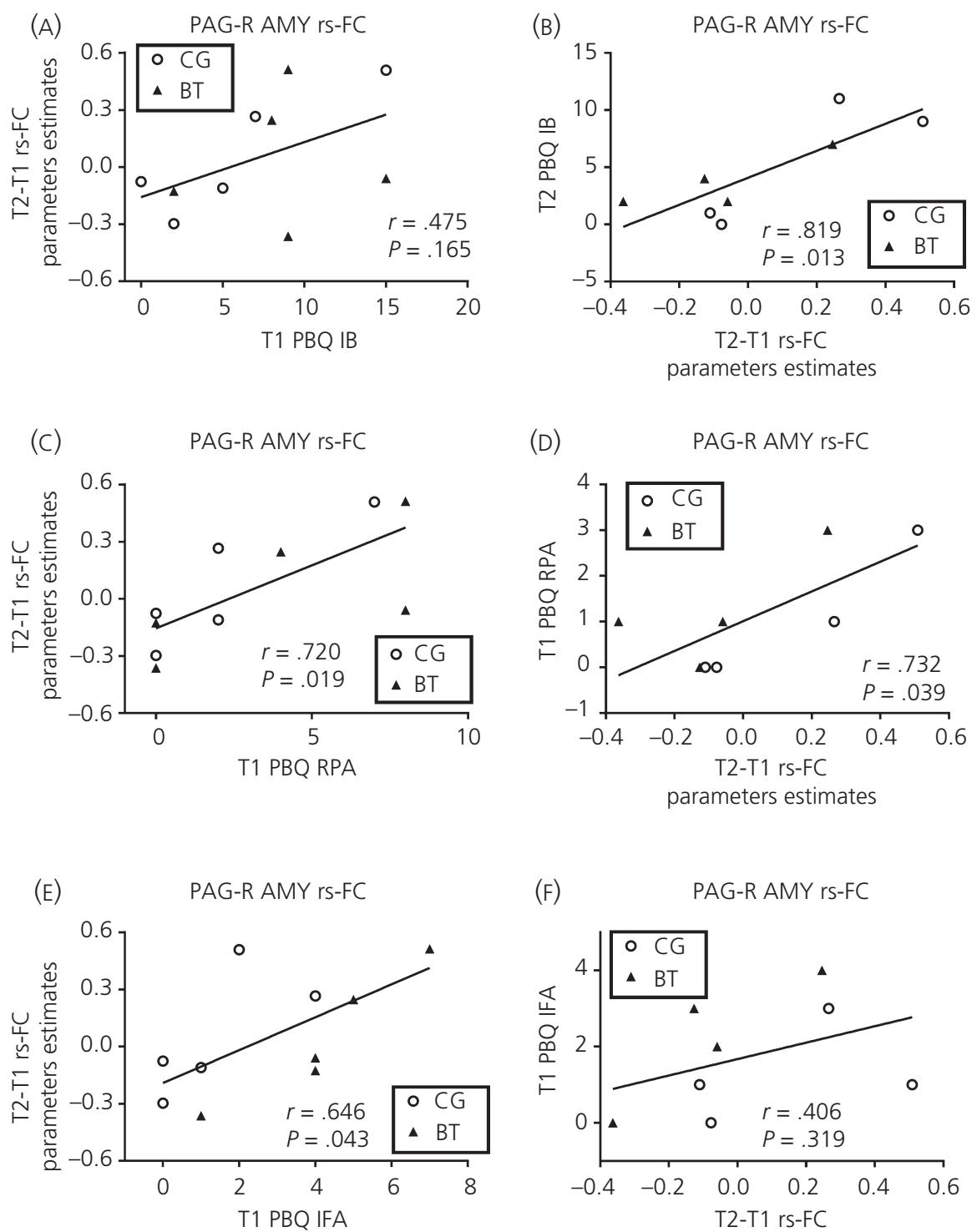

(F)

PAG-R AMY rs-FC

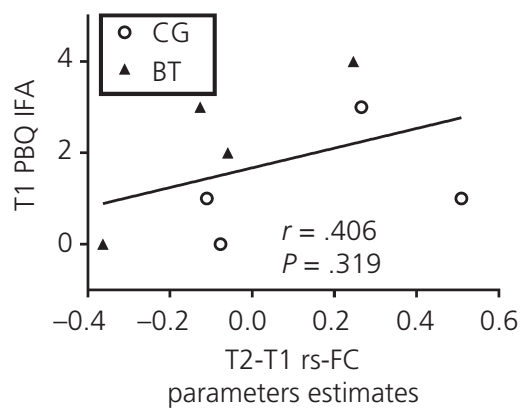

other. In the only rs-FC rodent study of the homologous maternal brain, connectivity between PAG and ACC was decreased for dams with early-life chronic social stress and interpreted as a decrease the reward salience of pups, resulting in depressed maternal care during early lactation. There was also an improvement in connectivity from early to late lactation (similar levels between groups during late lactation), which is considered to be the result of cumulative exposure to pup-related stimuli, such as that observed when nulliparous rats are induced to express maternal care by exposure to foster pups. ${ }^{111}$ Below, we discuss the possible maternal brain roles for regions that we report to have PAG-dependent rs-FC differences between BT and $\mathrm{CG}$ at $\mathrm{T} 1$ and $\mathrm{T} 2$.

Connectivity of PAG with other regions of the care and defence subsystems of the MBN to orchestrate parenting in humans is consistent with task-based $\mathrm{fMRI}$ responses to infant stimuli in the amygdala, ${ }^{58-60}$ insular cortex, ${ }^{61,62}$ OFC $^{63}$ and hypothalamus. ${ }^{64-66}$ In rodent studies, oxytocin neurones in the in mPOA of the hypothalamus regulate dopamine function in the ventral tegmental area and mediate the reward salience of pups. ${ }^{112,113}$ In another rat model, disrupted hypothalamus connectivity with PAG was related to impaired maternal care. ${ }^{114}$ Furthermore, rats that display low levels of maternal care exhibit lower oxytocin receptor binding in the mPOA of the hypothalamus, ${ }^{115}$ which strengthens the importance of this region with respect to regulating sensitive maternal care. The ACC also plays a key role in mediating appropriate responses to infant stimuli. In response to an infant cry, human mothers with lower depressive symptoms have increased activity in the dorsal ACC. ${ }^{116,117}$ Perhaps depression interferes with ACC-related empathy that has been demonstrated with human neuroimaging meta-analyses ${ }^{118,119}$ and parental brain studies. ${ }^{65,120}$ Furthermore, depressed mothers have been shown to exhibit decreases in the ACC-dependent rs-FC compared to healthy controls. ${ }^{76}$

The present study is preliminary and has notable limitations. First, given that the CG mothers were not affected by OUD at all and 
there was no placebo control, we could not control the influence of previous opioid use in confounding the effects of buprenorphine per se. Given the ethical and feasibility barriers to a placebo control trial with OUD subjects, future studies may be able to approach the pure effects of opioids with longitudinal research in which subjects are their own controls at different doses and time points. Future studies will also require a full characterisation of participants with OUD, including the quantity and frequency of all prescriptions, as well as licit and illicit drug, using a "time-line follow back" interview with calendar prompts and other memory aids to facilitate accurate recall. ${ }^{121-123}$ It will be also important to consider receptor occupancy as an important aspect of opioid dosing. Additional consideration will be required regarding the possible medical consequences of OUD, as well as sociodemographic and medical backgrounds, especially because we already know that parental stress, poverty, anxiety and postpartum depression affect the parental brain. ${ }^{80,124-126}$ Indeed, stress response dysregulation has been established in other substance use disorders. ${ }^{127-130}$ As noted in the Introduction, changes in rs-FC must be interpreted with caution because they do not capture the directionality of effect, and instead reflect basic associations of brain activity at rest, which are themselves under study Next, as a result of the small sample size in the samples with PBQ, the results with respect to testing the group difference in $\mathrm{PBQ}$ and the associations between the PBQ subscales and PAG-dependent rs-FC warrant replication in future studies. Furthermore, there are complex caveats around the measurement of parental thoughts and behaviours. In the present study, we only used the PBQ, which is a self-report measure that may not capture aspects of parenting about which participants may be embarrassed or unaware. Future work would benefit from more comprehensive interviews such as the working model of the child interview ${ }^{131}$ and parental interview of thoughts and behaviours, ${ }^{66,132}$ as well as video-based objective measures of parenting sensitivity, intrusiveness, interfering, forcing, overriding, anger/hostility and criticising of their child. ${ }^{133-135}$ Finally, the translational validity of the MBN across species requires more ethologically valid neuroimaging paradigms connected to real-world behavioural condition.

Although preliminary, the present study probes potential buprenorphine effects on mothers affected by OUD. BT is currently the best practice for pregnant women suffering OUD, despite the potential adverse effects of any exogenous opioids on maternal behaviour. It is therefore important to examine whether buprenorphine treatment for OUD, as a partial mu-opioid agonist and kappa-opioid antagonist, exerts beneficial or harmful effects on maternal brain and behaviour during the postpartum. On the one hand, our work may have identified a brain mechanism for the potential benefits of buprenorphine treatment with respect to reversing abnormality of maternal brain function and behaviour: the initial group differences in PAG-dependent resting-state functional connectivity had dissipated by 4 months postpartum, perhaps representing postpartum adjustments in this sample of mothers with healthy infants who were receiving ongoing medical care. On the other hand, our work may have identified a potential link between excessive PAG-hypothalamus functional connectivity and bonding impairments, for which the effects of buprenorphine remain undetermined. In the future, this work may be combined with brain models of other conditions that interfere with parenting, such as parental stress, poverty, anxiety and postpartum depression, ${ }^{80,124-126}$ as well as potential brain mechanisms at work in parenting interventions, ${ }^{136}$ to inform and optimise comprehensive interventions for BT mothers and fathers and identify the potential links between parental brain function and child outcomes. ${ }^{137}$ In sum, our preliminary work calls for more attention to PAG-dependent functional connectivity in the MBN as a possible brain mechanism for better assessing opioid-sensitive parental brain functions in the context of parenting behaviours and parent-child bonding.

\section{CONFLICT OF INTERESTS}

The authors declare that have no conflicts of interest.

\section{DATA AVAILABILITY STATEMENT}

The data that support the findings of this study are available from the corresponding author upon reasonable request.

\section{REFERENCES}

1. Krans EE, Patrick SW. Opioid use disorder in pregnancy: health policy and practice in the midst of an epidemic. Obstet Gynecol. 2016;128:4-10

2. Haight S, Ko J, Tong V, Bohm M, Callaghan W. Opioid use disorder documented at delivery hospitalization - United States, 19992014. MMWR Morb Mortal Wkly Rep. 2018;845-849.

3. Organization $\mathrm{W}-\mathrm{WH}$. Guidelines for the Identification and Management of Substance Use and Substance Use Disorders in Pregnancy. Geneva, Switzerland: WHO Press; 2014.

4. Nanda S, Brant R, Regier M, Yossuck P. Buprenorphine: a new player in neonatal withdrawal syndrome. WV Med J. 2015;111:16-21.

5. Krans EE, Bogen D, Richardson G, Park SY, Dunn SL, Day N. Factors associated with buprenorphine versus methadone use in pregnancy. Subst Abus. 2016;37:550-557.

6. Zedler BK, Mann AL, Kim MM, et al. Buprenorphine compared with methadone to treat pregnant women with opioid use disorder: a systematic review and meta-analysis of safety in the mother, fetus and child. Addiction. 2016;111:2115-2128.

7. McCarthy JJ, Leamon MH, Finnegan LP, Fassbender C. Opioid dependence and pregnancy: minimizing stress on the fetal brain. Am J Obstet Gynecol. 2017;216:226-231.

8. Bachhuber MA, Mehta PK, Faherty LJ, Saloner B. Medicaid coverage of methadone maintenance and the use of opioid agonist therapy among pregnant women in specialty treatment. Med Care. 2017;55:985-990.

9. Kaltenbach K, Berghella V, Finnegan L. Opioid dependence during pregnancy. Effects and management. Obstet Gynecol Clin North Am. 1998;25:139-151.

10. Whitten L. Buprenorphine during pregnancy reduces neonate distress.NIDA notes. http://www.drugabuse.gov/news-events/nida-notes/2012/07/buprenorphine-during-pregnancyreduces-neonate-distress: NIH-NIDA 2012. June, 2019.

11. Kakko J, Heilig M, Sarman I. Buprenorphine and methadone treatment of opiate dependence during pregnancy: comparison of fetal growth and neonatal outcomes in two consecutive case series. Drug Alcohol Depend. 2008;96:69-78. 
12. Johnson S, Martin PR. Transitioning from methadone to buprenorphine maintenance in management of opioid use disorder during pregnancy. Am J Drug Alcohol Abuse. 2018;44:310-316

13. Meyer MC, Johnston AM, Crocker AM, Heil SH. Methadone and buprenorphine for opioid dependence during pregnancy: a retrospective cohort study. J Addict Med. 2015;9:81-86.

14. Gaalema DE, Scott TL, Heil SH, et al. Differences in the profile of neonatal abstinence syndrome signs in methadone- versus buprenorphine-exposed neonates. Addiction. 2012;107(Suppl 1):53-62.

15. Hall ES, Isemann BT, Wexelblatt SL, et al. A cohort comparison of buprenorphine versus methadone treatment for neonatal abstinence syndrome. J Pediatr. 2016;170:39-44.

16. Mucke S, Nagel M, Siedentopf J, Buhrer C, Huseman D. Neonatal abstinence syndrome: twelve years of experience at a regional referral center. Klin Padiatr. 2017;229:32-39.

17. Lemon LS, Caritis SN, Venkataramanan R, Platt RW, Bodnar LM. Methadone versus buprenorphine for opioid use dependence and risk of neonatal abstinence syndrome. Epidemiology. 2017;29:261-268.

18. Brogly SB, Saia KA, Walley AY, Du HM, Sebastiani P. Prenatal buprenorphine versus methadone exposure and neonatal outcomes: systematic review and meta-analysis. Am J Epidemiol. 2014;180:673-686.

19. Lund IO, Fitzsimons H, Tuten M, Chisolm MS, O'Grady KE, Jones $\mathrm{HE}$. Comparing methadone and buprenorphine maintenance with methadone-assisted withdrawal for the treatment of opioid dependence during pregnancy: maternal and neonatal outcomes. Subst Abuse Rehabil. 2012;3(Suppl 1):17-25.

20. Jones $\mathrm{HE}$, Heil SH, Baewert A, et al. Buprenorphine treatment of opioid-dependent pregnant women: a comprehensive review. Addiction. 2012;107(Suppl 1):5-27.

21. Cleary BJ, Donnelly JM, Strawbridge JD, et al. Methadone and perinatal outcomes: a retrospective cohort study. Am J Obstet Gynecol. 2011;204:139.

22. Unger A, Jagsch R, Bawert A, et al. Are male neonates more vulnerable to neonatal abstinence syndrome than female neonates? Gend Med. 2011;8(6):355-364.

23. Fischer G, Ortner R, Rohrmeister K, et al. Methadone versus buprenorphine in pregnant addicts: a double-blind, double-dummy comparison study. Addiction. 2006;101:275-281.

24. Kayemba-Kay's S, Laclyde JP. Buprenorphine withdrawal syndrome in newborns: a report of 13 cases. Addiction. 2003;98:1599-1604.

25. Schindler SD, Eder $\mathrm{H}$, Ortner R, Rohrmeister $\mathrm{K}$, Langer $\mathrm{M}$, Fischer G. Neonatal outcome following buprenorphine maintenance during conception and throughout pregnancy. Addiction. 2003;98:103-110.

26. Jones $\mathrm{HE}$, Johnson RE, Jasinski DR, et al. Buprenorphine versus methadone in the treatment of pregnant opioid-dependent patients: effects on the neonatal abstinence syndrome. Drug Alcohol Depend. 2005;79:1-10.

27. Jones HE, Kaltenbach $\mathrm{K}$, Heil SH, et al. Neonatal abstinence syndrome after methadone or buprenorphine exposure. N Engl J Med. 2010;363:2320-2331.

28. Laslo J, Brunner JM, Burns D, et al. An overview of available drugs for management of opioid abuse during pregnancy. Matern Health Neonatol Perinatol. 2017;3:4.

29. Salihu HM, Salinas A, Medina I, Krishnaswami J, Aliyu MH. Biopsychosocial determinants of opioid use disorder (OUD) and implications for maternal and child health research: a scoping review. J Opioid Manag. 2019;15:77-91.

30. Kim S, lyengar U, Mayes LC, Potenza MN, Rutherford HJV, Strathearn L. Mothers with substance addictions show reduced reward responses when viewing their own infant's face. Hum Brain Mapp. 2017;38:5421-5439.
31. Swain JE, Ho SS, Fox H, Garry D, Brummelte S. Effects of opioids on the parental brain in health and disease. Front Neuroendocrinol. 2019;May 22:100766. https://doi.org/10.1016/j. yfrne.2019.100766. [Epub ahead of print].

32. Barth RP. Preventing child abuse and neglect with parent training: evidence and opportunities. Future Child. 2009;19:95-118.

33. Young NK, Boles SM, Otero C. Parental substance use disorders and child maltreatment: overlap, gaps, and opportunities. Child Maltreat.. 2007;12(2):137-149.

34. Slamberova R, Szilagyi B, Vathy I. Repeated morphine administration during pregnancy attenuates maternal behavior. Psychoneuroendocrinology. 2001;26:565-576.

35. Bridges RS, Grimm CT. Reversal of morphine disruption of maternal behavior by concurrent treatment with the opiate antagonist naloxone. Science. 1982;218:166-168.

36. Grimm CT, Bridges RS. Opiate regulation of maternal behavior in the rat. Pharmacol Biochem Behav. 1983;19:609-616.

37. Mann PE, Kinsley CH, Bridges RS. Opioid receptor subtype involvement in maternal behavior in lactating rats. Neuroendocrinology. 1991;53:487-492.

38. Rubin BS, Bridges RS. Disruption of ongoing maternal responsiveness in rats by central administration of morphine sulfate. Brain Res. 1984;307:91-97.

39. Stafisso-Sandoz G, Polley D, Holt E, Lambert KG, Kinsley $\mathrm{CH}$. Opiate disruption of maternal behavior: morphine reduces, and naloxone restores, c-fos activity in the medial preoptic area of lactating rats. Brain Res Bull. 1998;45:307-313.

40. Sukikara MH, Mota-Ortiz SR, Baldo MV, Felício LF, Canteras NS. A role for the periaqueductal gray in switching adaptive behavioral responses. J Neurosci. 2006;26:2583-2589.

41. Klein MO, Cruz Ade M, Machado FC, Picolo G, Canteras NS, Felicio LF. Periaqueductal gray mu and kappa opioid receptors determine behavioral selection from maternal to predatory behavior in lactating rats. Behav Brain Res. 2014;274:62-72.

42. Weinshenker NJ, Siegel A. Bimodal classification of aggression: affective defense and predatory attack. Aggress Violent Beh. 2002;7:237-250.

43. Numan M, Young LJ. Neural mechanisms of mother-infant bonding and pair bonding: similarities, differences, and broader implications. Horm Behav. 2016;77:98-112.

44. Numan M. A neural circuitry analysis of maternal behavior in the rat. Acta Paediatr Suppl. 1994;397:19-28.

45. Numan M. Motivational systems and the neural circuitry of maternal behavior in the rat. Dev Psychobiol. 2007:49:12-21.

46. Numan M. Hypothalamic neural circuits regulating maternal responsiveness toward infants. Behav Cogn Neurosci Rev. 2006;5:163-190.

47. Numan $M$, Sheehan TP. Neuroanatomical circuitry for mammalian maternal behavior. Ann N Y Acad Sci. 1997;807:101-125.

48. Numan M, Woodside B. Maternity: neural mechanisms, motivational processes, and physiological adaptations. Behav Neurosci. 2010;124:715-741.

49. Numan M, Numan MJ. Projection sites of medial preoptic area and ventral bed nucleus of the stria terminalis neurons that express Fos during maternal behavior in female rats. J Neuroendocrinol. 1997;9:369-384.

50. Stack EC, Balakrishnan R, Numan MJ, Numan M. A functional neuroanatomical investigation of the role of the medial preoptic area in neural circuits regulating maternal behavior. Behav Brain Res. 2002;131:17-36.

51. Swain JE, Ho SS. Neuroendocrine mechanisms for parental sensitivity: overview, recent advances and future directions. Curr Opin Psychol. 2017;15-105-110.

52. Bornstein $M H$, Putnick DL, Rigo $P$, et al. Neurobiology of culturally common maternal responses to infant cry. Proc Natl Acad Sci USA. 2017;114:E9465-E9473. 
53. Kim $P$, Strathearn L, Swain JE. The maternal brain and its plasticity in humans. Horm Behav. 2016;77:113-123.

54. Feldman R. The neurobiology of mammalian parenting and the biosocial context of human caregiving. Horm Behav. 2016;77:3-17.

55. Laurent HK, Stevens A, Ablow JC. Neural correlates of hypothalamic-pituitary-adrenal regulation of mothers with their infants. Biol Psychiatry. 2011;70:826-832.

56. Hipwell AE, Guo C, Phillips ML, Swain JE, Moses-Kolko EL. Right frontoinsular cortex and subcortical activity to infant cry is associated with maternal mental state talk. J Neurosci. 2015;35:12725-12732.

57. Swain JE, Mayes LC, Leckman JF. The development of parent-infant attachment through dynamic and interactive signaling loops of care and cry. Behav Brain Sci. 2004;27:472-473.

58. Riem MME, Bakermans-Kranenburg MJ, van ljzendoorn MH, Out $D$, Rombouts S. Attachment in the brain: adult attachment representations predict amygdala and behavioral responses to infant crying. Attach Human Dev. 2012;14:533-551.

59. Riem MM, van IJzendoorn IMH, Tops M, Boksem MA, Rombouts SA, Bakermans-Kranenburg MJ. No laughing matter: intranasal oxytocin administration changes functional brain connectivity during exposure to infant laughter. Neuropsychopharmacology 2012;37:1257-1266.

60. Riem MM, Bakermans-Kranenburg MJ, van IJzendoorn IMH. Intranasal administration of oxytocin modulates behavioral and amygdala responses to infant crying in females with insecure attachment representations. Attach Hum Dev 2016;18:213-234.

61. Elmadih A, Wan MW, Downey D, Elliott R, Swain JE, Abel KM. Natural variation in maternal sensitivity is reflected in maternal brain responses to infant stimuli. Behav Neurosci. 2016;130:500-510.

62. Riem MM, Bakermans-Kranenburg MJ, Pieper S, et al. Oxytocin modulates amygdala, insula, and inferior frontal gyrus responses to infant crying: a randomized controlled trial. Biol Psychiatry. 2011;70:291-297.

63. Swain JE, Kim P, Ho SS. Neuroendocrinology of parental response to baby-cry. J Neuroendocrinol. 2011;23:1036-1041.

64. Strathearn L, Fonagy P, Amico J, Montague PR. Adult attachment predicts maternal brain and oxytocin response to infant cues. Neuropsychopharmacology. 2009;34:2655-2666.

65. Ho SS, Konrath S, Brown S, Swain JE. Empathy and stress related neural responses in maternal decision making. Front Neurosci. 2014;8:152.

66. Swain JE, Tasgin E, Mayes LC, Feldman R, Constable RT, Leckman JF. Maternal brain response to own baby-cry is affected by cesarean section delivery. J Child Psychol Psychiatry. 2008;49:1042-1052.

67. Rutherford HJV, Potenza MN, Mayes LC. The neurobiology of addiction and attachment. In: Suchman N, Pajulo M, Mayes LC, eds. Parenting and Substance Abuse: Developmental Approaches to Intervention. New York, NY: Oxford University Press; 2013:3-23.

68. Rutherford HJ, Mayes LC. Parenting and addiction: neurobiological insights. Curr Opin Psychol. 2017;1555-1560.

69. Biswal BB. Resting state fMRI: a personal history. Neurolmage. 2012;62:938-944.

70. Buckner RL, Krienen FM, Yeo BT. Opportunities and limitations of intrinsic functional connectivity MRI. Nat Neurosci. 2013;16:832-837.

71. Ugurbil K. What is feasible with imaging human brain function and connectivity using functional magnetic resonance imaging. Philos Trans R Soc Lond B Biol Sci. 2016;371:pii: 20150361.

72. Cole DM, Smith SM, Beckmann CF. Advances and pitfalls in the analysis and interpretation of resting-state FMRI data. Front Syst Neurosci. 2010;4:8.

73. Rosazza C, Minati L. Resting-state brain networks: literature review and clinical applications. Neurol Sci. 2011;32:773-785.
74. Sripada RK, Swain JE, Evans GW, Welsh RC, Liberzon I. Childhood poverty and stress reactivity are associated with aberrant functional connectivity in default mode network. Neuropsychopharmacology. 2014;39:2244-2251.

75. Nephew BC, Febo M, Huang W, et al. Early life social stress and resting state functional connectivity in postpartum rat anterior cingulate circuits. J Affect Disord. 2018;229:213-223.

76. Deligiannidis KM, Sikoglu EM, Shaffer SA, et al. GABAergic neuroactive steroids and resting-state functional connectivity in postpartum depression: a preliminary study. J Psychiatr Res. 2013;47:816-828.

77. Chase HW, Moses-Kolko EL, Zevallos C, Wisner KL, Phillips ML. Disrupted posterior cingulate-amygdala connectivity in postpartum depressed women as measured with resting BOLD fMRI. Soc Cogn Affect Neurosci. 2014;9:1069-1075.

78. Mars RB, Neubert FX, Noonan MP, Sallet J, Toni I, Rushworth MF. On the relationship between the "default mode network" and the "social brain". Front Hum Neurosci. 2012;6:189.

79. Johnson MK, Raye CL, Mitchell KJ, Touryan SR, Greene EJ, Nolen-Hoeksema S. Dissociating medial frontal and posterior cingulate activity during self-reflection. Soc Cogn Affect Neurosci. 2006;1:56-64.

80. Moses-Kolko EL, Horner MS, Phillips ML, Hipwell AE, Swain JE. In search of neural endophenotypes of postpartum psychopathology and disrupted maternal caregiving. J Neuroendocrinol. 2014;26:665-684.

81. Brockington IF, Fraser C, Wilson D. The Postpartum Bonding Questionnaire: a validation. Arch Womens Ment Health. 2006;9:233-242.

82. Klier CM. Mother-infant bonding disorders in patients with postnatal depression: the Postpartum Bonding Questionnaire in clinical practice. Arch Womens Ment Health. 2006;9:289-291.

83. Ohara M, Okada T, Kubota $\mathrm{C}$, et al. Validation and factor analysis of mother-infant bonding questionnaire in pregnant and postpartum women in Japan. BMC Psychiatry. 2016;16:212.

84. Ohashi Y, Kitamura T, Sakanashi K, Tanaka T. Postpartum bonding disorder: factor structure, validity, reliability and a model comparison of the postnatal bonding questionnaire in Japanese mothers of infants. Healthcare. 2016;4:pii: E50.

85. Reck C, Klier CM, Pabst K, et al. The German version of the Postpartum Bonding Instrument: psychometric properties and association with postpartum depression. Arch Womens Ment Health. 2006;9:265-271.

86. Conway KP, Compton W, Stinson FS, Grant BF. Lifetime comorbidity of DSM-IV mood and anxiety disorders and specific drug use disorders: results from the National Epidemiologic Survey on Alcohol and Related Conditions. J Clin Psychiatry. 2006;67:247-257.

87. Davis MA, Lin LA, Liu H, Sites BD. Prescription opioid use among adults with mental health disorders in the United States. J Am Board Fam Med. 2017;30:407-417.

88. Murray L, Cooper PJ. Postpartum Depression and Child Development. New York, NY: Guilford Press; 1997.

89. Righetti-Veltema M, Bousquet A, Manzano J. Impact of postpartum depressive symptoms on mother and her 18-month-old infant. Eur Child Adolesc Psychiatry. 2003;12:75-83.

90. McLearn KT, Minkovitz CS, Strobino DM, Marks E, Hou W. Maternal depressive symptoms at 2 to 4 months post partum and early parenting practices. Arch Pediatr Adolesc Med. 2006;160:279-284.

91. Hay DF, Pawlby S, Waters CS, Sharp D. Antepartum and postpartum exposure to maternal depression: different effects on different adolescent outcomes. J Child Psychol Psychiatry. 2008;49:1079-1088.

92. Grace SL, Evindar A, Stewart DE. The effect of postpartum depression on child cognitive development and behavior: a review 
and critical analysis of the literature. Arch Womens Ment Health. 2003;6:263-274.

93. Halligan SL, Murray L, Martins C, Cooper PJ. Maternal depression and psychiatric outcomes in adolescent offspring: a 13-year longitudinal study. J Affect Disord. 2007;97:145-154.

94. Goodman SH, Rouse MH, Connell AM, Broth MR, Hall CM Heyward D. Maternal depression and child psychopathology: a meta-analytic review. Clin Child Fam Psychol Rev. 2011;14:1-27.

95. Netsi E, Pearson RM, Murray L, Cooper P, Craske MG, Stein A. Association of persistent and severe postnatal depression with child outcomes. JAMA Psychiatry. 2018;75:247-253.

96. Feldman R, Granat A, Pariente C, Kanety H, Kuint J, GilboaSchechtman E. Maternal depression and anxiety across the postpartum year and infant social engagement, fear regulation, and stress reactivity. J Am Acad Child Adolesc Psychiatry. 2009;48:919-927.

97. Brockington IF, Oates J, George S, et al. A screening questionnaire for mother-infant bonding disorders. Arch Womens Ment Health. 2001;3:133-140.

98. Jenkinson M, Bannister P, Brady M, Smith S. Improved optimization for the robust and accurate linear registration and motion correction of brain images. Neurolmage. 2002;17:825-841.

99. Ashburner J. A fast diffeomorphic image registration algorithm. Neurolmage. 2007;38:95-113.

100. Power JD, Barnes KA, Snyder AZ, Schlaggar BL, Petersen SE. Spurious but systematic correlations in functional connectivity MRI networks arise from subject motion. Neurolmage. 2012;59:2142-2154.

101. Fair DA, Nigg JT, lyer S, et al. Distinct neural signatures detected for ADHD subtypes after controlling for micro-movements in resting state functional connectivity MRI data. Front Syst Neurosci. 2012;6:80.

102. Behzadi Y, Restom K, Liau J, Liu TT. A component based noise correction method (CompCor) for BOLD and perfusion based fMRI. Neurolmage. 2007;37:90-101.

103. Chai XJ, Castanon AN, Ongur D, Whitfield-Gabrieli S. Anticorrelations in resting state networks without global signal regression. Neurolmage. 2012;59:1420-1428.

104. Ezra M, Faull OK, Jbabdi S, Pattinson KT. Connectivity-based segmentation of the periaqueductal gray matter in human with brainstem optimized diffusion MRI. Hum Brain Mapp. 2015;36:3459-3471.

105. Harricharan S, Rabellino D, Frewen PA, et al. fMRI functional connectivity of the periaqueductal gray in PTSD and its dissociative subtype. Brain Behav. 2016;6:e00579.

106. Eklund A, Nichols TE, Knutsson H. Cluster failure: why fMRI inferences for spatial extent have inflated false-positive rates. Proc Natl Acad Sci USA. 2016;113:7900-7905.

107. Smith SM, Nichols TE. Threshold-free cluster enhancement: addressing problems of smoothing, threshold dependence and localisation in cluster inference. Neurolmage. 2009;44:83-98.

108. Bartels A, Zeki S. The neural correlates of maternal and romantic love. Neurolmage. 2004;21(3):1155-1166.

109. Noriuchi M, Kikuchi Y, Senoo A. The functional neuroanatomy of maternal love: mother's response to infant's attachment behaviors. Biol Psychiatry. 2008;63:415-423.

110. Noriuchi M, Kikuchi Y, Mori K, Kamio Y. The orbitofrontal cortex modulates parenting stress in the maternal brain. Sci Rep. 2019;9:1658.

111. Bridges RS, Russell DW. Steroidal interactions in the regulation of maternal behaviour in virgin female rats: effects of testosterone, dihydrotestosterone, oestradiol, progesterone and the aromatase inhibitor, 1,4,6-androstatriene-3,17-dione. J Endocrinol. 1981;90:31-40.
112. Shahrokh DK, Zhang TY, Diorio J, Gratton A, Meaney MJ. Oxytocin-dopamine interactions mediate variations in maternal behavior in the rat. Endocrinology. 2010;151:2276-2286.

113. Lippard ET, Jarrett TM, McMurray MS, et al. Early postpartum pup preference is altered by gestational cocaine treatment: associations with infant cues and oxytocin expression in the MPOA. Behav Brain Res. 2015;278:176-185.

114. Fodor A, Klausz B, Pinter O, et al. Maternal neglect with reduced depressive-like behavior and blunted c-fos activation in Brattleboro mothers, the role of central vasopressin. Horm Behav. 2012;62:539-551.

115. Francis DD, Champagne FC, Meaney MJ. Variations in maternal behaviour are associated with differences in oxytocin receptor levels in the rat. J Neuroendocrinol. 2000;12:1145-1148.

116. Laurent HK, Ablow JC. A cry in the dark: depressed mothers show reduced neural activation to their own infant's cry. Soc Cogn Affect Neurosci. 2012;7:125-134.

117. Laurent HK, Ablow JC. The missing link: mothers' neural response to infant cry related to infant attachment behaviors. Infant Behav Dev. 2012;35:761-772.

118. Fan $Y$, Duncan NW, de Greck M, Northoff G. Is there a core neural network in empathy? An fMRI based quantitative meta-analysis. Neurosci Biobehav Rev. 2011;35:903-911.

119. Lamm C, Decety J, Singer T. Meta-analytic evidence for common and distinct neural networks associated with directly experienced pain and empathy for pain. Neurolmage. 2011;54:2492-2502.

120. Strathearn L, Li J, Fonagy P, Montague PR. What's in a smile? Maternal brain responses to infant facial cues. Pediatrics. 2008;122:40-51.

121. Carey KB. Reliability and validity of the time-line follow-back interview among psychiatric outpatients: a preliminary report. Psychol Addict Behav. 1997;11:26-33.

122. Sobell MB, Breslin FC, Sobell LC. Project MATCH: the time has come ... to talk of many things. J Stud Alcohol. 1998;59:124-125.

123. Sobell LC, Sobell MB, Leo GI, Cancilla A. Reliability of a timeline method: assessing normal drinkers' reports of recent drinking and a comparative evaluation across several populations. $\mathrm{Br} J$ Addict. 1988;83:393-402.

124. Ho SS, Swain JE. Depression alters maternal extended amygdala response and functional connectivity during distress signals in attachment relationship. Behav Brain Res. 2017;325:290-296.

125. Kim P, Ho SS, Evans GW, Liberzon I, Swain JE. Childhood social inequalities influences neural processes in young adult caregiving. Dev Psychobiol. 2015;57:948-960.

126. Guo C, Moses-Kolko E, Phillips M, Swain JE, Hipwell AE. Severity of anxiety moderates the association between neural circuits and maternal behaviors in the postpartum period. Cogn Affect Behav Neurosci. 2018;18:426-436.

127. Fox HC, Sinha R. Sex differences in drug-related stress-system changes: implications for treatment in substance-abusing women. Harv Rev Psychiatry. 2009;17:103-119.

128. Fox HC, Bergquist KL, Peihua G, Rajita S. Interactive effects of cumulative stress and impulsivity on alcohol consumption. Alcohol Clin Exp Res. 2010;34:1376-1385.

129. Sinha R, Fox HC, Hong KI, Hansen J, Tuit K, Kreek MJ. Effects of adrenal sensitivity, stress- and cue-induced craving, and anxiety on subsequent alcohol relapse and treatment outcomes. Arch Gen Psychiatry. 2011;68:942-952.

130. Fox HC, Milivojevic V, Angarita GA, Stowe R, Sinha R. Peripheral immune system suppression in early abstinent alcohol-dependent individuals: links to stress and cue-related craving. J Psychopharmacol. 2017;31:883-892.

131. Benoit D, Parker KC, Zeanah $\mathrm{CH}$. Mothers' representations of their infants assessed prenatally: stability and association with 
infants' attachment classifications. J Child Psychol Psychiatry. 1997;38:307-313.

132. Kim P, Mayes L, Feldman R, Leckman JF, Swain JE. Early postpartum parental preoccupation and positive parenting thoughts: relationship with parentinfant interaction. Infant Ment Health J. 2013;34:104-116.

133. Feldman R. Mother-newborn Coding System Manual. Tel Aviv, Israel: Bar-Ilan University Press; 1998.

134. Ferber SG, Feldman R, Makhoul IR. The development of maternal touch across the first year of life. Early Hum Dev. 2008;84:363-370.

135. Feldman R, Weller A, Sirota L, Eidelman Al. Testing a family intervention hypothesis: the contribution of mother-infant skin-to-skin contact (kangaroo care) to family interaction, proximity, and touch. J Fam Psychol. 2003;17:94-107.

136. Swain JE, Ho SS, Rosenblum KL, Morelen D, Dayton CJ, Muzik M. Parent-child intervention decreases stress and increases maternal brain activity and connectivity during own baby-cry: an exploratory study. Dev Psychopathol. 2017;29:535-553.

137. Kim $\mathrm{P}$, Rigo $\mathrm{P}$, Leckman JF, et al. A prospective longitudinal study of perceived infant outcomes at 18-24 months: neural and psychological correlates of parental thoughts and actions assessed during the first month postpartum. Front Psychol. 2015;6:17-72.

How to cite this article: Swain JE, Ho SS. Early postpartum resting-state functional connectivity for mothers receiving buprenorphine treatment for opioid use disorder: A pilot study. J Neuroendocrinol. 2019;31:e12770. https://doi. org/10.1111/jne.12770 